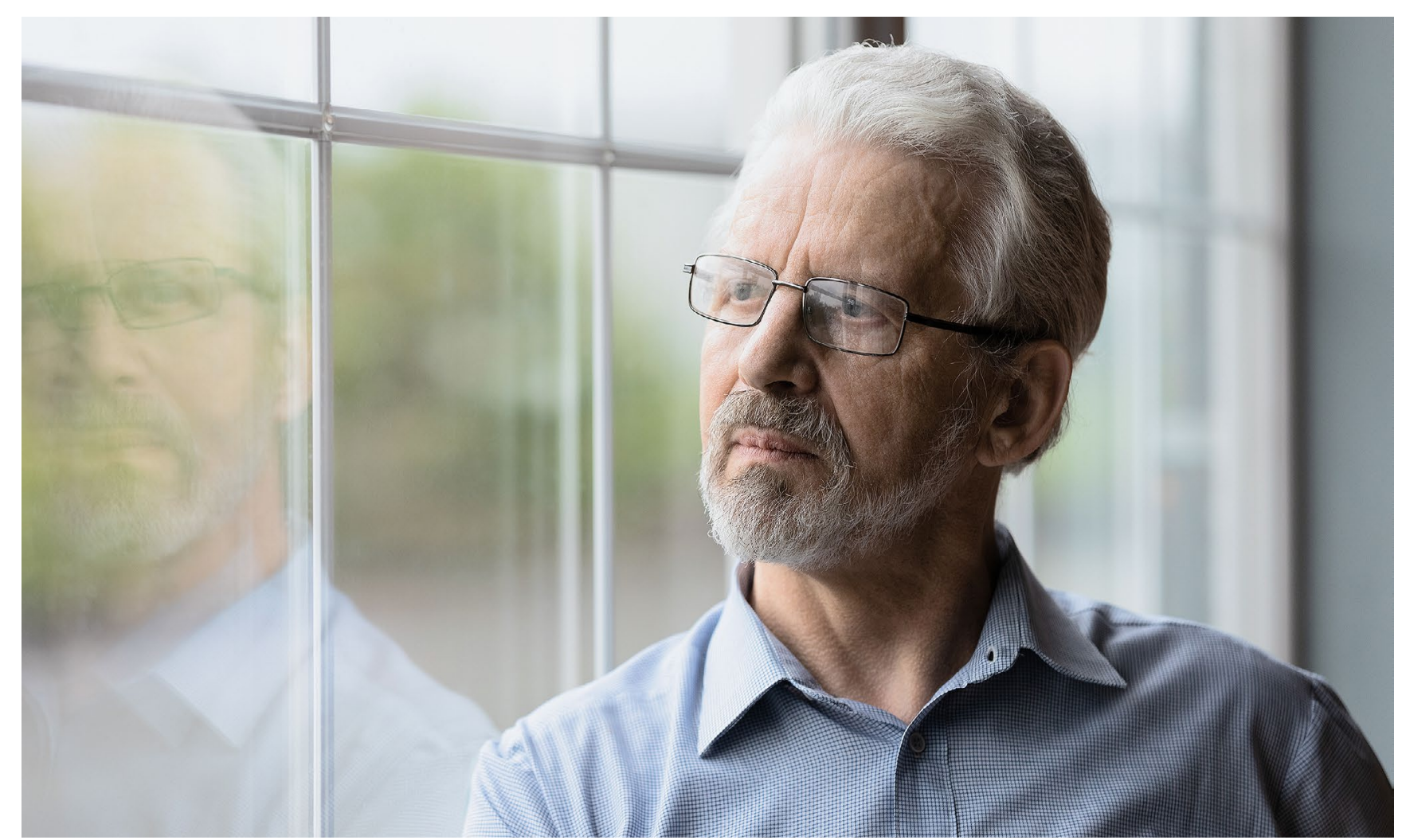

\title{
Das Long-Covid-Syndrom
}

\section{Neue Leitlinie mit Behandlungsempfehlungen für die Primärversorgung-- Autor: M. Bleckwenn}

Die Langzeitfolgen von COVID-19 rücken immer stärker in den Fokus. Experten aus 21 Fachgesellschaften, Organisationen und Institutionen haben unter Führung der Deutschen Gesellschaft für Pneumologie (DGP) in rekordverdächtiger Zeit - von Ende März bis Mitte Juli - eine S1-Leitlinie Post-COVID/Long-COVID ausgearbeitet und publiziert. Im Folgenden werden die für Hausärzte und Internisten wichtigsten Punkte skizziert.

Für Deutschland registrierte das Robert-KochInstitut (RKI) bisher etwa 4 Millionen Infektionen mit SARS-CoV-2. Schon früh gab es Meldungen zu möglichen Langzeitfolgen einer Corona-Infektion. Jedoch ist das Ausmaß der Problematik weiterhin noch nicht abschätzbar. Experten gehen bisher davon aus, dass etwa jeder zehnte Covid-Patient behandlungsbedürftige Symptome über einen längeren Zeitraum entwickelt. Nach der inzwischen eineinhalb Jahren währenden Coronapandemie würden damit etwa 400.000 Menschen in Deutschland unter Long-Covid leiden.
Bei ca. 45.0000 Hausärzten in Deutschland müsste jeder Hausarzt im Durchschnitt etwa zehn LongCovid-Patienten behandeln. Dies wäre verglichen mit anderen Erkrankungen eine verschwindend geringe Anzahl an Betroffenen. Allerdings benötigen Patienten mit Long-Covid zum Teil eine komplexe und multimodale Therapie. Zudem ist der weitere Verlauf der Pandemie nicht absehbar.

Der beste Schutz vor Long-Covid ist die Impfung gegen SARS-CoV-2. In Deutschland sind bisher etwa $60 \%$ der Bevölkerung vollständig geimpft. Daher könnte die Anzahl an Long-Covid-Patienten 


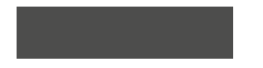

Es gibt

keinen

Laborpara-

meter, mit

dem man

Long-COVID

ein- oder

ausschließen

könnte.

\subsection{0}

Menschen leiden in Deutschland schätzungsweise unter Langzeitfolgen von COVID-19. im Verlauf des nächsten Winters noch deutlich ansteigen.

Die erste Anlaufstelle für die meisten Patienten ist der Hausarzt. Bisher gibt es jedoch keine spezifische Therapie gegen die Langzeitfolgen einer Covid-Infektion. Im Juli dieses Jahres erschien unter der Federführung der Deutschen Gesellschaft für Pneumologie die erste Leitlinie für Post-COVID/Long-COVID [1]. Dabei handelt es sich um eine S1-Leitlinie, also um Empfehlungen auf Basis eines informellen Konsenses. Dieser Beitrag fasst die Teile der Leitlinie zusammen, die für die hausärztliche Behandlung besonders relevant erscheinen.

\section{Definition Long-Covid- / Post-Covid-19- Syndrom}

Wenn der Patient 4 Wochen nach der akuten Infektion weiterhin über Beschwerden klagt, die im Zusammenhang mit der Covid-19-Infektion stehen, spricht man von Long-Covid. Dies können Beschwerden sein, die seit der Infektion bestehen oder auch nach Abklingen der akuten Beschwerden neu hinzugekommen sind. So kann sich im Verlauf der Erkrankung das Symptombild immer wieder verändern. Bestehen die Symptome länger als 12 Wochen und sind nicht durch eine andere Diagnose erklärbar, bezeichnet man dies als Post-COVID-19-Syndrom [2]. Die Gründe für die länger anhaltenden Beschwerden scheinen multifaktoriell zu sein und können sich bei jedem Patienten unterschiedlich ausprägen. Eine Ursache könnte eine monatelange Viruspersistenz sein, die wiederum zu anhaltenden Gewebeschäden und zu einer längeren Immunreaktion führt.

\section{Häufige Beschwerden: Fatigue, Kopfschmerz, Husten und depressive Verstimmung}

Wenn Patienten über persistierende Beschwerden nach einer Covid-19-Infektion klagen oder neue Beschwerden im Zusammenhang mit der Infektion hinzugekommen sind, kann Long-Covid bzw. ein PostCovid-19-Syndrom vorliegen (zur Vereinfachung wird im weiteren Beitrag von Long-Covid gesprochen). Häufig geäußerte körperliche Beschwerden sind unter anderem Fatigue, Leistungseinschränkung, Kopfschmerzen und Husten. Im Verlauf der Erkrankung kommen gehäuft psychische Beschwerden wie depressive Verstimmung und Angstsymptomatik hinzu. Aber auch kardiale Beschwerden wie Palpitation und Thoraxschmerzen können die Patienten beeinträchtigen. Bei dem Erstkontakt mit dem Patienten ist es wichtig, andere Erkrankungen, die zu diesen Beschwerden führen können, auszuschließen.

\section{Anamnese und körperliche Untersuchung}

Aufgrund der Komplexität des Krankheitsbilds ist eine ausführliche Anamnese und körperliche Unter- suchung bei diesen Patienten notwendig. Die Anamnese umfasst neben dem zeitlichen Verlauf der Beschwerden auch die soziale, familiäre und berufliche Situation. Zudem sollte erfragt werden, welche neuen funktionellen Einschränkungen durch die Covid-Infektion eingetreten sind. Anschließend erfolgt eine körperliche Untersuchung einschließlich der Erhebung eines neurologischen und psychiatrischen Status. Dabei sollten auch der Blutdruck, die Herzfrequenz und die Temperatur bestimmt werden. Neben der Atemfrequenz sollte, wenn technisches Gerät vorhanden, zusätzlich die Sauerstoffsättigung gemessen werden. Um den funktionellen Status einschätzen zu können, kann ein validierter Fragebogen eingesetzt werden (Tab. 1).

\section{Diagnostik}

Die Leitlinie empfiehlt bei allem Long-Covid-Patienten ein Basislabor (BB, CRP, Kreatinin, Harnstoff, Transaminasen, TSH und Urin-Stix) abzunehmen. Weitere Parameter müssen nur bei spezifischen Beschwerden bzw. Erkrankungen, wie bei kardiovaskulären Komplikationen, bestimmt werden. Insgesamt gibt es keinen Laborparameter, mit dem man Long-Covid ein- oder ausschließen könnte. Die weitere Basisdiagnostik orientiert sich in der Leitlinie nach den geäußerten Beschwerden des Patienten. Zur weiteren Diagnostik und Therapie können Patienten, insbesondere bei pathologischen Befunden, in eine Post-Covid-Ambulanz überwiesen werden. Dabei haben die Ambulanzen unterschiedliche Schwerpunkte wie Neurologie, Pulmologie oder Kardiologie. Im Folgenden wird die Diagnostik bei den beiden sehr häufigen Long-Covid-Symptomen beschrieben:

\section{Dyspnoe/ thorakale Beschwerden}

Bei einer anhaltenden Dyspnoe nach einer CovidErkrankung sollten eine Lungenfunktion und ein EKG durchgeführt werden. Zudem ist die Anfertigung eines Röntgen-Thorax und die Bestimmung der D-Dimere zu überlegen. Durch Fragen nach der Belastbarkeit kann die Schwere der Erkrankung eingeordnet werden. Zur genaueren Abklärung von unklarer Dyspnoe ist dann eine lungenfachärztliche Abklärung notwendig. Dies umfasst ein Funktionstest in Ruhe, wie die Messung der Diffusionskapazität und eine Blutgasanalyse sowie ein Funktionstest unter Belastung, wie ein 6-Minuten-Gehtest oder eine Ergospirometrie. Bei pathologischen Befunden in der Diagnostik sollte im Anschluss eine Computertomografie des Thorax durchgeführt werden.

Neben den pulmonalen Ursachen der Dyspnoe bzw. thorakalen Beschwerden kommt auch eine kardiale Genese in Betracht. Die Patienten haben je nach Schweregrad der Akuterkrankung ein deutlich 


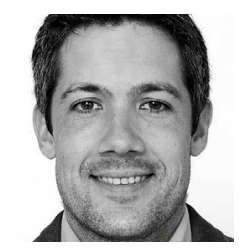

Prof. Dr. med.

Markus Bleckwenn

Selbstständige

Abteilung für

Allgemeinmedizin,

Medizinische

Fakultät der

Universität Leipzig

E-Mail:

Markus.Bleckwenn@

medizin.uni-leipzig.de

Literatur:

als Zusatzmaterial unter springermedizin./mmw

Die Verordnung
von Heilmitteln
im
Zusammenhang
mit Post-/
Long-Covid-19
belastet nicht
das
hausärztliche
Budget.

erhöhtes Risiko für kardiovaskuläre Komplikationen wie Schlaganfall, Myokardinfarkt oder Lungenembolie [3]. Daher sollte im Zweifel eine weiterführende kardiale Diagnostik mit einer Echokardiographie und ggf. einem Belastungs-EKG eingeleitet werden. Pathologischen Befunde in der Echokardiografie können dann mittels kardialem MRT weiter abgeklärt werden. Für eine routinemäßige Durchführung eines Kardio-MRT in der Post-Covid-19-Phase sieht die Leitlinie keine Indikation.

\section{Fatigue}

Die Patienten beschreiben eine subjektiv stark einschränkende Erschöpfung auf somatischer, kognitiver und/oder psychischer Ebene. Diese Erschöpfung verbessert sich nicht ausreichend durch Schonung oder Schlaf. Zunächst sollten differenzialdiagnostisch organische- oder psychische Erkrankungen ausgeschlossen werden. Screeningfragen können dabei erste Hinweise auf mögliche Begleiterkrankungen wie Depression oder Angststörung geben. Der Schweregrad der Erkrankung kann durch psychometrische Selbstauskunftsinstrumente, wie der Fatigue Schweregrad Skala (FSS), bestimmt werden. Bei Patienten unter 60 Jahren und einer schweren Fatigue sollte bei einer Beschwerdepersistenz für mehr als 6 Monate an das Vorliegen eines Chronischen Fatigue Syndroms (CFS) gedacht werden. Diese Patienten berichten neben einer Belastungsintoleranz über
Schmerzen und kognitive Einschränkungen. Patienten mit dem Verdacht auf ein CFS sollten zum Neurologen zur weiteren Abklärung überwiesen werden. Sollte ein CFS vorliegen, droht den Patienten eine Chronifizierung der Beschwerden mit einem zum Teil lebenslangen Verlust der Leistungsfähigkeit.

\section{Therapie}

Wenn sich in der primärärztlichen Basisdiagnostik keine Auffälligkeiten bzw. Warnhinweise für lebensbedrohliche Erkrankungen ergeben und die Beschwerden sich nicht verschlechtern, wird ein abwartendes Verhalten (watchful waiting) empfohlen. Zudem übernimmt der Hausarzt die Koordination einer ggf. notwendigen spezialisierten Behandlung. Bei Bedarf kann der Hausarzt den Patienten im Rahmen der psychosomatischen Grundversorgung und psychosozial betreuen. Bei der Begleitung von LongCovid kann dem Patienten die in der Regel günstige Prognose seiner Erkrankung vermittelt werden. Bisher gibt es keine belastbaren wissenschaftlichen Belege für eine spezifische Therapie von LongCovid. Daher wird allgemein eine symptomorientierte Therapie empfohlen. Im Folgenden werden Therapieansätze bei einzelnen Beschwerden aufgeführt. Bei persistierenden Hustenbeschwerden kann ein Inhalatives Corticosteroid $+/$ - Bronchodilatatoren als Therapieversuch eingesetzt werden. Zudem kann auch ein langwirksamer Muskarinantagonist

Tab. 1 Die Post-Covid-19-Skala zur Bestimmung des funktionellen Status im zeitlichen Verlauf nach einer Covid-Infektion (Post-COVID-19 Functional Status [PCFS]-Skala nach [4])

\section{Wie stark sind Sie gegenwärtig in Ihrem Alltag durch COVID-19 beeinträchtigt? \\ (Bitte geben Sie an, welche der folgenden \\ Aussagen am ehesten auf Sie zutrifft)}

Ich habe keine Einschränkungen in meinem Alltag und keine Symptome wie Schmerzen, Depressionen oder Ängste im Zusammenhang mit der Infektion.

Ich habe vernachlässigbare Einschränkungen in meinem Alltag, da ich alle üblichen Aufgaben/Aktivitäten ausführen kann, obwohl ich immer noch anhaltende Symptome, Schmerzen, Depressionen oder Ängste habe.

Ich leide unter Einschränkungen in meinem täglichen Leben, da ich gelegentlich gewohnte Pflichten/Aktivitäten aufgrund von Symptomen wie Schmerzen, Depressionen oder Angstzuständen vermeiden oder reduzieren muss. Ich bin jedoch in der Lage, alle Aktivitäten ohne Hilfe durchzuführen.

Ich leide unter Einschränkungen in meinem täglichen Leben, da ich nicht in der Lage bin, alle üblichen Aufgaben/Aktivitäten aufgrund von Symptomen wie Schmerzen, Depressionen oder Angstzustände durchzuführen. Ich bin jedoch in der Lage, mich selbst zu versorgen / bzw. ohne Hilfe für mich selbst zu sorgen.

Ich leide unter starken Einschränkungen in meinem Alltag: Ich bin aufgrund von Symptomen wie Schmerzen, Depressionen oder Angstzustände nicht in der Lage, mich selbst zu versorgen und bin daher auf Pflege und/oder Hilfe einer anderen Person angewiesen.

\section{Entsprechender \\ Grad auf der PCFS-Skala}

$0=$ keine

Funktionseinschränkung

$1=$ vernachlässigbare

Funktionseinschränkungen

2 = leichte funktionelle

Einschränkungen

$3=$ moderate funktionelle

Einschränkungen

$4=$ schwerwiegende funktionelle Einschränkungen 
(LAMA) versucht werden. Bei starken Beschwerden kann auch eine unterstützende Atem- und Physiotherapie hilfreich sein.

Bei eingeschränkten Körperfunktionen, Müdigkeit und Erschöpfung sollte wohldosiertes körperliches Training angeleitet werden. Bei Bedarf können die Patienten zusätzlich physiotherapeutisch betreut werden. Bei der Verordnung von Heilmitteln im Zusammenhang mit Post-Covid / Long-Covid kommt den Hausärzten zugute, dass diese bei einer Wirtschaftlichkeitsprüfung nicht das Budget belasten. Dazu muss bei der Verordnung die Diagnose „U09.9 Post-COVID-19-Zustand, nicht näher bezeichnet" kodiert werden. Zudem kann bei diesen Verordnungen von der Höchstmenge pro Rezept abgewichen werden. Neben der Physiotherapie kann auch eine ergotherapeutische Behandlung sinnvoll sein.

Patienten mit Fatigue müssen lernen, mit ihren persönlichen Ressourcen umzugehen (Coping). Sie sollen sich nicht überfordern, aber auch nicht Aktivitäten meiden. Eine körperliche Überbeanspruchung kann bei diesen Patienten die Symptome verschlechtern und dann zu einer Schonungsreaktion führen. Zudem kann eine psychotherapeutische und ggf. auch psychopharmakologische Behandlung notwendig sein.

Wenn die Long-Covid-Symptome in der ambulanten Behandlung persistieren oder sich verschlechtern, ist eine akutstationäre Therapie oder eine (teil-) stationäre Rehabilitation angezeigt. Im Anschluss sollte der weitere Rehabilitationsfortschritt und psychosozialer Unterstützungsbedarf regelmäßig durch den Hausarzt überprüft werden. Zudem kann der Patient in eine Selbsthilfe-Gruppe eingebunden werden.

\section{FAZIT FÜR DIE PRAXIS}

1. Long-Covid-Patienten benötigen zum Teil eine komplexe multimodale Therapie.

2. Der Hausarzt koordiniert die Behandlung von Patienten mit Long-Covid- / Post-Covid-Syndrom.

3. Bei allen Patienten mit dem Verdacht auf Long-Covid sollten mithilfe einer ausführlichen Anamnese und körperlichen Untersuchung sowie einer Basisdiagnostik andere Erkrankungen ausgeschlossen werden.

4. Wenn in der Basisdiagnostik keine Warnhinweise für einen gefährlichen Krankheitsverlauf erkennbar sind, wird ein abwartendes Verhalten empfohlen.

5. Bisher gibt es keine spezifische Therapie für anhaltende Beschwerden nach einer Covid-Infektion.

6. Bei Leistungseinschränkungen wird ein kontrolliertes Training empfohlen. 